\title{
Using the ribosome to synthesize peptidomimetics
}

\section{Roger M Freidinger}

Address: Independent Medicinal Chemistry Consultant, 744 Newport Lane, Lansdale, PA 19446, USA

Email: rfreidinger@comcast.net

Fl000 Biology Reports 2009, I:53 (doi:10.34I0/BI-53)

The electronic version of this article is the complete one and can be found at: http://FI000.com/Reports/Biology/content/I/53

\begin{abstract}
Peptidomimetic research is an approach to identify peptide-based drugs designed to mimic structural, conformational, and biological properties of peptides while overcoming their limitations, such as protease instability and poor cell penetration. With recent advances in ribosomal synthesis of peptides containing unnatural amino acids, this technology appears suitable for preparing large structurally diverse libraries of peptidomimetics for drug discovery screening.
\end{abstract}

\section{Introduction and context}

Biologically active peptides are attracting increasing interest as potential therapeutic agents or leads toward such agents [1]. For many applications, native peptides are not ideal due to inherent issues, including short biological half-life (rapid cleavage by proteases and/or fast clearance), poor membrane permeability that limits bioavailability and access to intracellular targets, and physical-chemical properties [2]. Research over the last several decades has focused on the discovery, design, and synthesis of novel structures that possess the desirable properties of native peptides while overcoming their liabilities $[3,4]$. These 'peptidomimetics' have originated from both chemical synthesis and natural product sources [5,6]. An emerging complementary technology providing access to amide backbone-based peptidomimetic structures is ribosomal synthesis using unnatural amino acids. The attractive potential of ribosomal synthesis is rapid and specific preparation of large structurally diverse libraries of peptidomimetics for screening against biological targets.

The history of this approach and key recent advances in the technology have been detailed in review articles $[7,8]$ in the last year and will be treated only briefly. Twenty years ago, two groups were able to reassign stop (or nonsense) codons to non-proteinogenic amino acids, and using chemically generated misaminoacylated transfer ribonucleic acid (tRNA), they demonstrated the incorporation of unnatural amino acids into a protein and a polypeptide $[9,10]$. This 'nonsense suppression' is suitable for the incorporation of only a single type of non-proteinogenic amino acid into a peptide chain. A complementary method using nucleotide four-base codons allowed the incorporation of two or three unnatural amino acids but is still limited in its generality. Other limitations of these methods are the restricted choice of unnatural amino acids that can be incorporated and the technically challenging process of chemically or enzymatically aminoacylating tRNA.

One key technical advance in the current decade was the development of a reconstituted Escherichia coli cell-free translation system (for example, protein synthesis using recombinant elements, or PURE [11]) in which certain amino acids and aminoacyl-tRNA synthetases (aaRSs) are removed from the translation elements. Specific vacant codons thus can be created and used for unnatural amino acids of choice. Lack of competition from the natural aminoacyl-tRNA thereby improves the efficiency of sense suppression, and the potential of this approach for production of large screenable libraries of small peptidomimetics was proposed [12]. It was emphasized that such directed in vitro evolution is much faster than lead optimization by chemical synthesis and that the superior library sizes may yield higher-affinity ligands. A second major advance came with the development of more general methods for the preparation of 
Figure I. Schematic examples of peptidomimetic modifications achieved by ribosomal synthesis

Neptidomimetic: One or more of $\mathrm{R} 1, \mathrm{R} 2, \mathrm{R} 3$ comes
from an unnatural amino acid
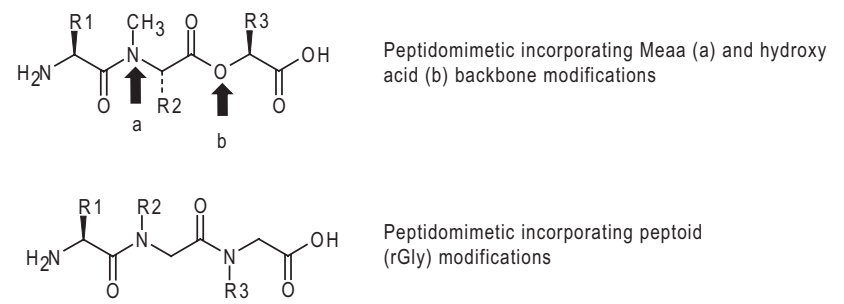

Cyclic peptidomimetics (circled amino acid can be one or multiple units)
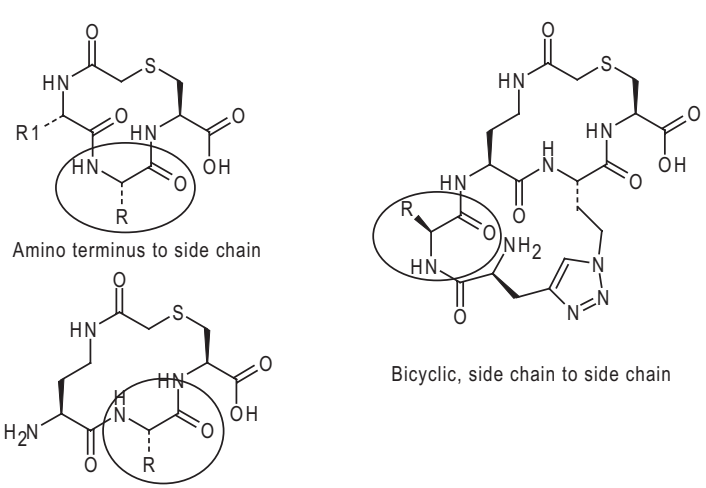

Bicyclic, side chain to side chain

Side chain to side chain

Ribosomal synthesis has produced peptidomimetics with considerable structural diversity. Examples include unnatural side chains, backbone modifications such as $\mathrm{N}$-methyl amino acids (Meaas) and hydroxy acids, and peptoids ( $N$-substituted glycine, or rGly). In addition, peptidomimetics incorporating such features can be cyclized. The macrocyclic rings can be formed by amino terminus-to-side chain or side chain-to-side chain cyclization, forming thioether bridges. Bicyclic peptides are synthesized using two pairs of orthogonally reactive groups to specifically locate the thioether and triazole bridges. The circled residues in the structures illustrate that a variety of ring sizes have been shown to be accessible.

nonstandard aminoacyl-tRNA. One of the most promising developments was the generation of artificial RNA enzymes (ribozymes) for tRNA aminoacylation. These so-called flexizymes [13] have a broad range of substrates (both tRNAs and amino acids), thus making available a general group of aminoacylated tRNAs. Recent publications have demonstrated that the use of one or both of these technologies for genetic code reprogramming is beginning to provide access to a diverse group of peptidomimetics (see Figure 1).

\section{Major recent advances}

One of the earliest investigations of ribosomal peptidomimetic synthesis used nonsense suppression to synthesize 16 amino acid peptides incorporating one unnatural amino acid [14]. Although limited in scope, this study was promising in that $N$-methyl-L-phenylalanine (MePhe) and L-phenyllactic acid (backbone modifications) were incorporated in addition to $\alpha$-amino acids with unusual side chains. Advances with reconstituted cell-free systems allowed the incorporation of three to five consecutive unnatural amino acids into a peptide sequence, although the structural scope was similar to that of the earlier studies $[12,15]$.

Further development of the PURE system for peptide synthesis allowed simultaneous reassignment of 35 of 61 sense codons to 12 unnatural amino acids that were previously known to be substrates for aaRSs and incorporated into proteins by E. coli [16]. Except for azetidine carboxylic acid, a backbone modification, these amino acids contain unnatural side chains spanning a range of physicochemical properties. The alkynyl side chain of 2-amino-hex-5-ynoic acid is of note, as it can undergo a copper (I)-catalyzed [3 + 2] cycloaddition reaction with small-molecule organic azides, leading to increased post-translational structural diversity [17]. A survey of additional unnatural amino acids showed that a diverse set of more than 50 amino acids can be incorporated into peptides by ribosomal translation [18]. Most of these are side-chain analogs, but also included are $\alpha, \alpha$-disubstituted and $N$-methyl amino acids (Meaas) and $\alpha$-hydroxy acids. With this reprogrammed genetic code, up to 13 different unnatural substitutions could be incorporated into a single peptidomimetic. This system potentially can synthesize unnatural peptide libraries of $10^{14}$ unique members.

Peptide backbone modifications such as Meaas and cyclization in peptidomimetic structures are useful for improving protease stability and membrane permeability and introducing conformational constraint [2]. A chemical reductive alkylation approach to overcoming limitations of scale in chemical aminoacylation preparation of misacylated tRNAs for ribosomal synthesis of Meaa-containing peptides was developed [19]. With this chemistry, the ribosomal synthesis of peptides containing up to three Meaas was demonstrated [20]. Of the Meaas surveyed, N-methyl leucine (MeLeu), $\mathrm{N}$-methyl threonine (MeThr), and $\mathrm{N}$-methyl valine (MeVal) were most efficiently incorporated into peptides. An alternative approach to a more efficient preparation of $\mathrm{N}$-methyl aminoacylated tRNA uses chemically synthesized $N$-methyl aminoacyl adenosine monophosphate (AMP) as the substrate for aaRSs [21]. Oligomerization of MePhe assigned to repeat consecutively two, five, or 10 times was demonstrated in an in vitro display system [22]. 
Another report expanded the study of amino acid backbone specificity of the E. coli translation machinery [23]. For the incorporation of alanine (Ala) and phenylalanine (Phe) analogs into the second position of a tripeptide, Meaas and hydroxy acids gave good yields, $\alpha, \alpha$-disubstituted amino acids incorporated poorly, and $\beta$ - and D-amino acids were not detectably incorporated. Further investigations showed that proper pairing of Meaas and tRNAs can be important for efficient expression, but the more bulky $N$-butyl-Ala and -Phe were not incorporated [24].

A very recent refinement in the technology for ribosomal synthesis of Meaa-containing peptides combines the PURE system, in which both selected amino acids and aaRSs are withdrawn (wPURE) to vacate certain codons, with flexizymes for charging unnatural amino acids onto desired tRNAs [25]. With this system, the N-methyl analogs of 11 of 19 natural plus four unnatural amino acids could be incorporated, and the preferences were somewhat different from those of previous studies. Peptides containing multiple Meaas were prepared.

In a significant advance, it was demonstrated that cyclic peptides incorporating Meaas could be synthesized by assigning an $N^{\alpha}$-( $\alpha$-chloroacetyl) amino acid to the initiation codon and a cysteine (Cys) to an elongation codon for a sequence [26]. Under the conditions of the experiment, the thiol of Cys displaces the $\alpha$-chloro in an intramolecular macrocyclization. Examples containing 27-atom rings with three or four Meaas or proline [25] plus examples with 15-, 21-, 36-, and 45-membered rings with natural amino acids [26] suggest considerable potential generality for this approach. Furthermore, the ribosomal synthesis of a library of 160 distinct peptides having 21-membered macrocycles was achieved. The macrocycle introduces an additional conformational constraint into the peptidomimetic, and the ability to synthesize libraries of such compounds for screening in biological assays is attractive.

The cyclization in these cases involves side chain-toamino terminus ring formation. A side chain-to-side chain variant has also been developed with the novel amino acid $N^{\gamma}$-(2-chloroacetyl)- $\alpha, \gamma$-diaminobutyric acid (Cab) [27]. This amino acid was efficiently incorporated using the wPURE and flexizyme technologies, and cyclization with Cys occurred under the experimental conditions giving examples with 20-, 23-, $26-$, and 35 -atom rings.

Further extension of this methodology has resulted in the ribosomal synthesis of bicyclic peptides involving two pairs of amino acids with orthogonal reactivity [28]. The pairs of amino acids are Cys and Cab and azidohomoalanine (Aha) and propargyl glycine ( $\mathrm{Pgl})$, respectively. As in previous cases, the first macrocyclic ring results from the formation of the thioether linkage under the translation reaction conditions. Copper (I) is then used to catalyze $[3+2]$ cycloaddition between the azide and alkyne side chains to form a triazole as the second bridge [17]. The preparation of several bicyclic peptidomimetic structures of different ring sizes serves to illustrate the versatility of the methodology.

Very recently, ribosomal synthesis of polypeptoids (poly- $N$-substituted glycines, or poly-rGlys) [29] and peptoid-peptide hybrids was reported [30]. A group of 25 rGlys containing a variety of groups was shown to be incorporated, although bulkiness near the $\alpha$-amino group and charged substituents do not work well. Consecutive rGlys could be incorporated, and cyclization strategies as previously discussed were also successful for peptoid-peptide hybrid sequences.

This technology has been extended recently to the ribosomal synthesis of polyesters [31] and to translation initiation with D-amino acids [32]. The latter advance allows for capping the amino terminus of a peptide sequence with a D-amino acid or acyl-D-amino acid.

\section{Future directions}

Recent studies have shown the potential for generating libraries of peptidomimetic structures of significant structural diversity using ribosomal synthesis. Testing of such libraries in high-throughput screens offers the potential for discovery of leads to novel therapeutic agents. This approach may be complementary to chemical synthesis of such structures in terms of ligand diversity, speed, and cost.

Certain questions remain to be addressed. How broad is the chemical structural space that can be accessed by these methods? How useful will such peptidomimetic structures be as leads to biological tools and therapeutic agents? Will the purity and integrity of the individual structures in libraries from ribosomal synthesis be sufficient? Can promising lead structures be readily amplified in scale for follow-up studies? Given the speed at which this field is developing and the creative approaches being applied, the answers to these questions should be forthcoming in the near future.

\section{Abbreviations}

aaRS, aminoacyl-transfer ribonucleic acid synthetase; Aha, azidohomoalanine; Ala, alanine; AMP, adenosine monophosphate; Cab, $N^{\gamma}$-(2-chloroacetyl)- $\alpha, \gamma$-diaminobutyric acid; Cys, cysteine; Meaa, $N$-methyl amino 
acid; MeLeu, N-methyl leucine; MePhe, N-methyl-Lphenylalanine; MeThr, N-methyl threonine; MeVal, N-methyl valine; Phe, phenylalanine; PURE, protein synthesis using recombinant elements; Pgl, propargyl glycine; rGly, N-substituted glycine; tRNA, transfer ribonucleic acid; wPURE, withdrawn protein synthesis using recombinant elements.

\section{Competing interests}

The author declares that he has no competing interests.

\section{References}

I. Stevenson CL: Advances in peptide pharmaceuticals. Curr Pharm Biotech 2009, I0:122-37.

2. Veber DF, Freidinger RM: The design of metabolically-stable peptide analogs. Trends Neurosci 1985, 8:392-6.

3. Ripka AS, Rich DH: Peptidomimetic design. Curr Opin Chem Biol 1998, 2:44|-52.

4. Freidinger RM: Nonpeptidic ligands for peptide and protein receptors. Curr Opin Chem Biol 1999, 3:395-406.

5. Walsh CT: Polyketide and nonribosomal peptide antibiotics: modularity and versatility. Science 2004, 303:1805-10.

6. Sieber SA, Maraheil MA: Learning from nature's drug factories: nonribosomal synthesis of macrocyclic peptides. J Bacteriol 2003, I 85:7036-43.

7. Kang TJ, Suga $\mathrm{H}$ : Ribosomal synthesis of nonstandard peptides. Biochem Cell Biol 2008, 86:92-9.

8. Ohta A, Yamagishi $Y$, Suga H: Synthesis of biopolymers using genetic code reprogramming. Curr Opin Chem Biol 2008, I2:159-67.

9. Noren CJ, Anthony-Cahill SJ, Griffith MC, Schultz PG: A general method for site-specific incorporaton of unnatural amino acids into proteins. Science 1989, 244:182-8.

10. Bain JD, Diala ES, Glabe CG, Dix TA, Chamberlin AR: Biosynthetic site-specific incorporation of a non-natural amino acid into a polypeptide. J Am Chem Soc 1989, I I I:8013-4.

II. Shimizu Y, Inoue A, Tomari Y, Suzuki T, Yokogawa T, Nishikawa K, Ueda T: Cell-free translation reconstituted with purified components. Nat Biotechnol 200I, 19:75I-5.

12. Forster AC, Tan Z, Nalam MN, Lin H, Qu H, Cornish WW, Blacklow SC: Programming peptidomimetic synthesis by translating genetic codes designed de novo. Proc Natl Acad Sci U S A 2003, 100:6353-7.

FI000 Factor 6.0 Must Read

Evaluated by Lee Gehrke 9 Sep 2003

13. Ohuchi M, Murakami H, Suga H: The flexizyme system: a highly flexible tRNA aminoacylation tool for the translation apparatus. Curr Opin Chem Biol 2007, I I:537-42.

14. Bain JD, Wacker DA, Kuo EE, Chamberlin AR: Site-specific incorporation of non-natural residues into peptides: effect of residue structure on suppression and translation efficiencies. Tetrahedron 1991, 47:2389-400.

15. Tan Z, Blacklow SC, Cornish VW, Forster AC: De novo genetic codes and pure translation display. Methods 2005, 36:279-90.

16. Josephson K, Hartman MCT, Szostak JW: Ribosomal synthesis of unnatural peptides. J Am Chem Soc 2005, I 27: I I727-35.

FI000 Factor 3.0 Recommended

Evaluated by Michael Famulok 30 Aug 2005
17. Kolb HC, Sharpless KB: The growing impact of click chemistry on drug discovery. Drug Discovery Today 2003, 8: I I 28-37.

18. Hartman MCT, Josephson K, Lin C-W, Szostak JW: An expanded set of amino acid analogs for the ribosomal translation of unnatural peptides. PLOS ONE 2007, 2:e972.

19. Merryman C, Green R: Transformation of aminoacyl tRNAs for the in vitro selection of 'drug-like' molecules. Chem Biol 2004, I I:575-82.

20. Subtelny AO, Hartman MCT, Szostak JW: Ribosomal synthesis of N-methyl peptides. J Am Chem Soc 2008, I30:6 I3I-6.

21. Sando S, Masu H, Furutani C, Aoyama Y: Enzymatic N-methylaminoacylation of tRNA using chemically misacylated AMP as a substrate. Org Biomol Chem 2008, 6:2666-8.

22. Frankel A, Millward SW, Roberts RW: Encodamers: unnatural peptide oligomers encoded in RNA. Chem Biol 2003, 10:1043-50.

FI000 Factor 3.0 Recommended Evaluated by Burckhard Seelig 19 Jan 2004

23. Tan Z, Forster AC, Blacklow SC, Cornish WW: Amino acid backbone specificity of the Escherichia coli translation machinery. J Am Chem Soc 2004, I26:12752-3.

24. Zhang B, Tan Z, Dickson LG, Nalam MNL, Cornish VW, Forster AC: Specificity of translation for $\mathbf{N}$-alkyl amino acids. J Am Chem Soc 2007, I 29: I |3 |6-7.

25. Kawakami T, Murakami H, Suga H: Messenger RNA-programmed incorporation of multiple $\mathbf{N}$-methyl-amino acids into linear and cyclic peptides. Chem Biol 2008, I 5:32-42.

FI000 Factor 6.0 Must Read

Evaluated by Niles Lehman 12 Feb 2008

26. Goto $Y$, Ohta A, Sako $Y$, Yamagishi $Y$, Murakami H, Suga H: Reprogramming the translation initiation for the synthesis of physiologically stable cyclic peptides. ACS Chem Biol 2008, 3:120-9.

FI000 Factor 3.2 Recommended

Evaluated by Dehua Pei OI Feb 2008, Ross Weatherman 20 Feb 2008

27. Sako $Y$, Goto $Y$, Murakami H, Suga H: Ribosomal synthesis of pepidase-resistant peptides closed by a nonreducible interside-chain bond. ACS Chem Biol 2008, 3:24I-9.

28. Sako $\mathrm{Y}$, Morimoto J, Murakami H, Suga H: Ribosomal synthesis of bicyclic peptides via two orthogonal inter-side-chain reactions. J Am Chem Soc 2008, 130:7232-4.

FI000 Factor 3.0 Recommended

Evaluated by Roger Freidinger 17 Jun 2008

29. Yoo B, Kirshenbaum K: Peptoid architectures: elaboration, actuation, and application. Curr Opin Chem Biol 2008, I 2:7|4-2I.

30. Kawakami T, Murakami $H$, Suga $H$ : Ribosomal synthesis of polypeptoids and peptoid-peptide hybrids. I Am Chem Soc 2008, I30:1686I-3.

31. Ohta A, Murakami H, Higashimura E, Suga H: Synthesis of polyester by means of genetic code reprogramming. Chem Biol 2007, 14:1315-22.

FI000 Factor 3.0 Recommended Evaluated by Scott K Silverman 23 Jan 2008

32. Goto $\mathrm{Y}$, Murakami H, Suga H: Initiating translation with D-amino acids. RNA 2008, | 4:1390-8. 